\title{
Diagnostic yield of endoscopic ultrasound-guided tissue acquisition for small solid pancreatic lesions
}

\section{다)(요 $\odot$}

\author{
Authors \\ Takafumi Mie, Takashi Sasaki, Ryo Kanata, Takaaki Furukawa, Tsuyoshi Takeda, Akiyoshi Kasuga, Masato \\ Matsuyama, Masato Ozaka, Naoki Sasahira
}

Institution

Cancer Institute Hospital of Japanese Foundation for Cancer Research, Department of Gastroenterology Center, Division of Hepato-Biliary-Pancreatic Medicine, Tokyo, Japan

submitted 20.3.2020

accepted after revision 25.5 .2020

\section{Bibliography}

Endoscopy International Open 2020; 08: E1359-E1364

DOI 10.1055/a-1230-3555

ISSN 2364-3722

(C) 2020. The Author(s).

This is an open access article published by Thieme under the terms of the Creative Commons Attribution-NonDerivative-NonCommercial License, permitting copying and reproduction so long as the original work is given appropriate credit. Contents may not be used for commecial purposes, or adapted, remixed, transformed or built upon. (https://creativecommons.org/licenses/by-nc-nd/4.0/)

\section{Corresponding author}

Takashi Sasaki, Cancer Institute Hospital of Japanese Foundation for Cancer Research, Department of Gastroenterology Center, Division of Hepato-BiliaryPancreatic Medicine, 3-8-31 Ariake, Koto-ku, Tokyo 135-8550, Japan

Fax: +81335200141

sasakit-tky@umin.ac.jp

\section{ABSTRACT}

Background and study aims Endoscopic ultrasound (EUS)-guided tissue acquisition is sometimes required to diagnose small solid pancreatic lesions. The aim of this study was to evaluate the diagnostic yield of EUS-guided tissue acquisition for small solid pancreatic lesions and the differences in diagnostic yield among different needles.

Patients and method We retrospectively analyzed consecutive patients who had undergone EUS-guided tissue acquisition for solid pancreatic lesions less than $2 \mathrm{~cm}$ between November 2012 and June 2019. Three types of needles were evaluated in this study: a 22-gauge fine-needle aspiration (FNA) Lancet needle, a 20-gauge fine-needle biopsy (FNB) Menghini needle with a lateral forward bevel, and a 22-gauge FNB Franseen needle. We evaluated the diagnostic yield and safety of the procedure using these needles.

Results We analyzed 160 patients with 163 lesions. The overall sensitivity, specificity, and accuracy were $92.0 \%$, $100 \%$, and $92.6 \%$, respectively. In the histological plus cytological diagnosis, accuracies of the Lancet, Menghini, and Franseen needles were 92.7\%, 97.0\%, and $85.7 \%$, respectively $(P=0.10)$. In the histological diagnosis alone, the negative predictive values (NPVs) of the Lancet, Menghini, and Franseen needles were 13.3\%, 53.3\%, and $27.3 \%$, respectively $(P=0.08)$. Adverse events occurred in four cases $(2.5 \%)$ : one postprocedural bleeding, two cases of pancreatitis, and one pancreatic abscess.

Conclusions EUS-guided tissue acquisition for small solid pancreatic lesions has a high diagnostic yield and safety. This study suggested a difference in the diagnostic yield of each needle for small solid pancreatic lesions.

\section{Introduction}

In 1992, Vilmann et al. was the first to report endoscopic ultrasound (EUS)-guided fine-needle aspiration (FNA) for pancreatic lesions [1]. To date, EUS-FNA has been established as a technique with high diagnostic performance in pancreatic lesions. Many studies have reported that EUS-FNA has high accuracy $(83 \%-95 \%)$ in detecting pancreatic lesions [2-4]. However, EUS-FNA can sometimes yield only small amounts of tissue from pancreatic lesions, which is insufficient for histologic diagnosis. Recently, several fine-needle biopsy (FNB) needles have become commercially available and widely used for tissue acquisition.

There are many kinds of solid pancreatic lesions, such as invasive ductal carcinoma, acinar cell neoplasms, neuroendocrine neoplasms, metastatic pancreatic tumors, and benign tumors. As a result of progress in diagnostic imaging techniques, many solid pancreatic lesions can be accurately diagnosed without 
histological evidence. However, especially for small pancreatic lesions, there are several cases in which the diagnosis is still difficult to confirm only by diagnostic imaging. Furthermore, such small pancreatic lesions are being found more frequently than ever due to recent advances in imaging modalities [5]. Because surgery for pancreatic lesions is highly invasive, it is very important to differentiate benign lesions (e.g., mass forming pancreatitis) from malignant lesions so as not to accidentally operate on benign tumors.

There are limited data on the diagnostic yield of EUS-guided tissue acquisition for small solid pancreatic lesions. Moreover, small lesions of the pancreas are sometimes difficult to puncture, and there are many unclear aspects about what type of needle is more suitable. Therefore, we conducted a retrospective study to evaluate the diagnostic yield of EUS-guided tissue acquisition for solid pancreatic lesions less than $2 \mathrm{~cm}$ in diameter and the difference in diagnostic yield among different needles.

\section{Patients and methods}

\section{Patients}

We retrospectively analyzed consecutive patients with solid pancreatic lesions less than $2 \mathrm{~cm}$ in diameter who underwent EUS-guided tissue acquisition at our institution between November 2012 and June 2019. Excluded patients were those who had received EUS-guided tissue acquisition by several types of needles or in several lesions in one session and did not receive individual diagnoses for each needle or each lesion and those who were lost to follow-up or who had not been followed up for at least 6 months in cases of benign tumors. Written informed consent for the procedure was obtained from all patients in accordance with the Declaration of Helsinki. This study was approved by the ethics committee of our institution.

\section{Procedure}

EUS-guided tissue acquisition was performed with a convex linear-array echoendoscope (GF-UCT260; Olympus Medical Systems, Tokyo, Japan) under analgesia and moderate sedation. All procedures were performed in hospitalized management. Three types of needles were used in more than 10 cases during this study period: (1) a 22G Expect Endoscopic Ultrasound Aspiration Needle (Boston Scientific Corp) (fine-needle aspiration [FNA] Lancet needle); (2) a $20 \mathrm{G}$ Echo Tip ProCore HD Ultrasound Biopsy Needle (Cook Endoscopy) (FNB Menghini needle) with a lateral forward bevel; and (3) a 22G Acquire Endoscopic Ultrasound Fine Needle Biopsy Device (Boston Scientific Corp) (FNB Franseen needle). These three needles were used properly according to the date of the procedure. In particular, the Lancet needle was mainly used between November 2012 and June 2016, the Menghini needle between July 2016 and January 2019, and the Franseen needle between February 2019 and June 2019. The procedure was performed by experts ( $\geq 5$ years of EUS-tissue acquisition experience) or trainees ( $<5$ years of EUS-tissue acquisition experience) under the guidance of experts.
Solid pancreatic lesions were detected endosonographically, and the maximum diameter of the lesion and the puncture diameter were measured. We defined the maximum diameter of the lesion as the size of the lesion. At first puncture, $10-\mathrm{mL}$ syringe suction was applied, and 20 strokes were performed. When we use the Menghini needle, we detected the side hole endosonographically, and stroke so that the side hole remains inside the lesion. To prevent mixture of the samples, we washed the lumen of the needle with saline and wiped the needle tip and stylet thoroughly with an alcohol swab after each puncture. When there was a large amount of blood to suction, the slow-pull method was used for the subsequent session at the discretion of the operator [6]. After each puncture, the sample in the needle was pushed into a Petri dish with saline, and rapid on-site evaluation (ROSE) was performed by the cytologist. When enough specimens were confirmed by ROSE, we finished the procedure with one additional puncture to acquire tissue sufficient for cytological and histological diagnosis, if we could puncture the lesion safely. Finally, the remaining specimens were fixed in $7 \%$ formalin and processed for histological examination. Immunohistochemical examination was performed, if necessary, at the discretion of the pathologists. We did not tell the cytologists and pathologists which needle was used.

\section{Final diagnosis}

For operated lesions, the final diagnosis was made by histological assessment of the resected specimen. For unresectable lesions, the final diagnosis was made by cytological or histological assessment of the tissue obtained by EUS-guided acquisition, and the subsequent clinical course was checked to confirm the consistency. In patients without pathological proof of malignancy, a final diagnosis of "benign" was made after at least 6 months of follow-up without evidence of progression on interval imaging.

\section{Evaluation}

This study evaluated the diagnostic yield of EUS-guided tissue acquisition and adverse events (AEs). Diagnostic yield was evaluated per lesion, while AEs were calculated per patient. When the same needle was used to puncture multiple lesions, each lesion was evaluated individually. If multiple needles were used for one lesion, the first needle yield was evaluated. Tissue acquisition rate was defined as the sum of lesions in which histological diagnosis could be performed divided by the total number of lesions. Diagnostic accuracy was defined as positive findings of malignancy with a final diagnosis of a malignant disease (true positive) or negative findings of malignancy with a final diagnosis of a benign disease (true negative). Diagnostic accuracy was defined as the sum of true-positive and true-negative results divided by the total number of lesions. We evaluated the physical examinations after the procedure, and blood tests and physical examinations were also evaluated at the first outpatient clinic after discharge. AEs were defined as any postprocedural events using a lexicon for endoscopic AEs [7]. 
172 patients, 176 lesions

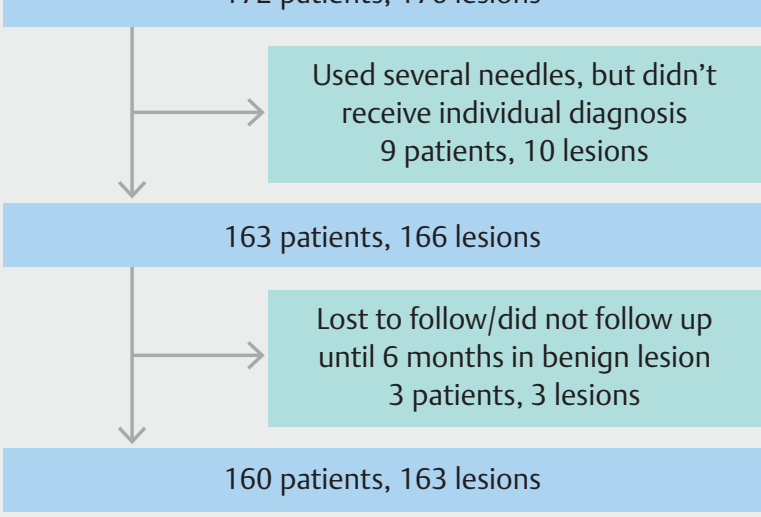

Fig. 1 Study flowchart.

\section{Statistical analysis}

Continuous variables are presented as medians (ranges) and were compared using the Kruskal-Wallis test. Categorical variables were described as absolute numbers (proportions) and were analyzed using the chi-squared or Fisher's exact test. $P<$ 0.05 was considered statistically significant. Statistical analyses were performed using EZR ver1.40 (Saitama Medical Center, Jichi Medical University, Saitama, Japan) [8]. The clinical data were followed up until August 2019.

\section{Results}

One hundred seventy-two patients with 176 solid pancreatic lesions less than $2 \mathrm{~cm}$ in diameter among them underwent EUSguided tissue acquisition during this period. Nine patients with 10 lesions underwent acquisition by several types of needles and did not receive individual diagnoses for each needle. One patient with one lesion was lost to follow-up and two patients with two lesions had not been followed up at 6 months in case of benign tumors. According to the exclusion criteria, the above patients were excluded. Therefore, 160 patients and 163 lesions were analyzed in this study ( $\mathbf{F i g} \mathbf{1} \mathbf{1}$ ). There were three patients who had two lesions punctured by the same needle. All lesions could technically be punctured by each needle. $>$ Table 1 shows characteristics of the lesions in this study. The tumor locations consisted of 48 lesions in the pancreatic head (29.4\%) and 115 in the pancreatic body/tail (70.6\%). Tmedian lesion size was 17 $\mathrm{mm}$ (range 8-20), and median puncture diameter was $15 \mathrm{~mm}$ (range $7-20$ ). The final diagnoses were 150 malignant lesions (129 pancreatic adenocarcinomas, 11 metastatic pancreatic tumors, seven neuroendocrine neoplasms, one solid pseudopapillary tumor, two other invasive cancers of the pancreas) and 13 benign lesions ( 8 cases of mass-forming pancreatitis and 5 benign pancreatic lesions). The punctures were performed through the stomach in 127 cases (77.9\%), the duodenum in 34 cases $(20.9 \%)$ and the jejunum in two cases $(1.2 \%)$. Median number of punctures was two (range 1-6).
- Table 1 Characteristics and final diagnosis of study lesions $(n=163)$.

\begin{tabular}{|c|c|}
\hline \multicolumn{2}{|l|}{ Location, n (\%) } \\
\hline - Pancreatic head & $48(29.4)$ \\
\hline - Pancreatic body/tail & $115(70.6)$ \\
\hline \multicolumn{2}{|l|}{ Access route, $\mathrm{n}(\%)$} \\
\hline - Stomach & $127(77.9)$ \\
\hline - Duodenum & $34(20.9)$ \\
\hline - Jejunum & $2(1.2)$ \\
\hline Number of punctures, $\mathrm{n}$ (range) & $2(1-6)$ \\
\hline Size of lesion, mm (range) & $18(8-20)$ \\
\hline Diameter of puncture, mm (range) & $15(7-20)$ \\
\hline \multicolumn{2}{|l|}{ Final diagnosis, n (\%) } \\
\hline - Pancreatic adenocarcinoma & $129(79.1)$ \\
\hline - Metastatic pancreatic tumor & $11(6.7)$ \\
\hline - Neuroendocrine neoplasm & $7(4.3)$ \\
\hline - Solid pseudopapillary neoplasm & $1(0.6)$ \\
\hline - Other cancer invasion to pancreas & $2(1.2)$ \\
\hline - Mass-forming pancreatitis & $8(4.9)$ \\
\hline - Benign pancreatic lesion & $5(3.1)$ \\
\hline
\end{tabular}

The 22G Lancet needles were used for 55 lesions, the $20 \mathrm{C}$ Menghini needles were used for 66, and the 22G Franseen needles were used for 42 . There was no significant difference in the location and size of the lesions or the access route among the three needles. The number of punctures was significantly higher and the median size and diameter of the punctures were significantly longer in the Menghini group than in the other two groups (number of punctures, $P<0.01$; lesion size, $P=0.03$; lesion diameter, $P<0.01)$. In the Menghini group, there was a trend toward fewer pancreatic adenocarcinomas than in the other groups. Tissue acquisition rate of the Lancet group was lower than the other two groups $(P<0.01)$ ( $>$ Table 2$)$.

Overall diagnostic yield was as follows: sensitivity $92.0 \%$, specificity $100 \%$, positive predictive value (PPV) $100 \%$, negative predictive value (NPV) $52.0 \%$ and accuracy $92.6 \%$. The diagnostic yield of each needle was also analyzed for histological plus cytological diagnosis and histological diagnosis alone. For histological plus cytological diagnosis, the sensitivities of the Lancet, Menghini, and Franseen groups were 92.5\%, 96.6\%, and $84.6 \%$, the NPVs were $33.3 \%, 80.0 \%$, and $33.3 \%$, and the accuracies were $92.7 \%, 97.0 \%$, and $85.7 \%$, respectively. The accuracy of the Franseen group tended to be low $(P=0.10)$. On the other hand, for histological diagnosis alone, the sensitivities of the Lancet, Menghini, and Franseen groups were $75.5 \%$, $87.9 \%$, and $79.5 \%$, the NPVs were $13.3 \%, 53.3 \%$, and $27.3 \%$ and the accuracies were $76.4 \%, 89.4 \%$, and $81.0 \%$, respective- 
- Table 2 Characteristics and final diagnosis with each needle

\begin{tabular}{|c|c|c|c|c|}
\hline & Lancet & Menghini & Franseen & $P$ value \\
\hline & $n=55$ & $n=66$ & $n=42$ & \\
\hline \multicolumn{4}{|l|}{ Location, n (\%) } & 0.26 \\
\hline - Pancreatic head & $20(36.4)$ & $15(22.7)$ & $13(31.0)$ & \\
\hline - Pancreatic body/tail & $35(63.6)$ & $51(77.3)$ & $29(69.0)$ & \\
\hline \multicolumn{4}{|l|}{ Access route, $n(\%)$} & 0.97 \\
\hline - Stomach & $43(78.2)$ & $52(78.8)$ & $32(76.2)$ & \\
\hline - Duodenum & $11(20.0)$ & $13(19.7)$ & $10(23.8)$ & \\
\hline - Jejunum & $1(1.8)$ & $1(1.5)$ & 0 & \\
\hline Median number of punctures, $n$ (range) & $2(1-6)$ & $3(1-6)$ & $2(1-5)$ & $<0.01$ \\
\hline Median size of lesion, $n$ (range) & $17(8-20)$ & $18(10-20)$ & $17(11-20)$ & 0.03 \\
\hline Median diameter of puncture, $\mathrm{mm}$ (range) & $14(7-20)$ & $16(9-20)$ & $15(9-20)$ & $<0.01$ \\
\hline Tissue acquisition rate, $\%$ & 89.1 & 100 & 97.6 & $<0.01$ \\
\hline \multicolumn{4}{|l|}{ Final diagnosis, n (\%) } & 0.09 \\
\hline - Pancreatic adenocarcinoma & $45(81.8)$ & $48(72.7)$ & $36(85.7)$ & \\
\hline - Metastatic pancreatic tumor & $4(7.3)$ & $6(9.1)$ & $1(2.4)$ & \\
\hline - Neuroendocrine tumor & $1(1.8)$ & $4(6.1)$ & $2(4.8)$ & \\
\hline - Solid pseudopapillary neoplasm & $1(1.8)$ & 0 & 0 & \\
\hline - Other cancer invasive pancreas & $2(3.6)$ & 0 & 0 & \\
\hline - Mass-forming pancreatitis & 0 & $7(10.6)$ & $1(2.4)$ & \\
\hline - Benign pancreatic lesion & $2(3.6)$ & $1(1.5)$ & $2(4.8)$ & \\
\hline
\end{tabular}

- Table 3 Diagnostic yield of each needle in histological plus cytological diagnosis, and histological diagnosis alone.

\begin{tabular}{|c|c|c|c|c|c|c|c|c|}
\hline & \multicolumn{4}{|c|}{ Histological plus cytological diagnosis } & \multicolumn{4}{|c|}{ Histological diagnosis alone } \\
\hline & Lancet & Menghini & Franseen & $P$ value & Lancet & Menghini & Franseen & $P$ value \\
\hline & $n=55$ & $n=66$ & $n=42$ & & $n=55$ & $n=66$ & $n=42$ & \\
\hline Sensitivity & $92.5 \%$ & $96.6 \%$ & $84.6 \%$ & 0.24 & $75.5 \%$ & $87.9 \%$ & $79.5 \%$ & 0.11 \\
\hline Specificity & $100 \%$ & $100 \%$ & $100 \%$ & 1.00 & $100 \%$ & $100 \%$ & $100 \%$ & 1.00 \\
\hline PPV & $100 \%$ & $100 \%$ & $100 \%$ & 1.00 & $100 \%$ & $100 \%$ & $100 \%$ & 1.00 \\
\hline NPV & $33.3 \%$ & $80.0 \%$ & $33.3 \%$ & 0.15 & $13.3 \%$ & $53.3 \%$ & $27.3 \%$ & 0.08 \\
\hline Diagnostic accuracy & $92.7 \%$ & $97.0 \%$ & $85.7 \%$ & 0.10 & $76.4 \%$ & $89.4 \%$ & $81.0 \%$ & 0.16 \\
\hline
\end{tabular}

ly. The NPV of the Menghini group had a tendency to be high (NPV; $P=0.08$ ). The accuracy of histological diagnosis alone among the groups was not statistically significant $(P=0.16)$ ( $\triangleright$ Table 3). Immunohistochemical examinations were performed in 33 specimens (20.2\%). Twenty-nine specimens (90.6\%) successfully underwent immunohistochemical exami- nation among these specimens (Lancet 6/8, Menghini 18/20, Franseen 5/5; $P=0.45)$.

Four AEs occurred in total $(2.5 \%$; $4 / 160)$. One case of mild pancreatitis and one severe pancreatic abscess occurred in the Lancet group, one mild bleed occurred in the Menghini group, and one case of mild pancreatitis occurred in the Franseen group. In the case of pancreatic abscess, abdominal pain oc- 
curred 3 days after the procedure and gradually increased. Inflammation was exacerbated, and the pancreatic abscess was detected by computed tomography. The patient was hospitalized for over 10 days and needed antibiotic treatment.

\section{Discussion}

We performed a retrospective study to evaluate the diagnostic yield of EUS-guided tissue acquisition of small solid pancreatic lesions. Ali $\mathrm{A}$ et al. reported that the diagnostic yield of EUSFNA decreases as lesion size decreases [9]. In previous studies, sensitivities, specificities, and accuracies of EUS-guided tissue acquisition for small solid pancreatic lesions have been reported with ranges of $75.9 \%$ to $82 \%, 70.4 \%$ to $100 \%$, and $72.1 \%$ to $82 \%$, respectively $[4,9,10]$. This study had equal to or better results in terms of diagnostic yield compared to the previous report (where the sensitivity, specificity, and accuracy were $84.6 \%$ to $96.6 \%, 100 \%$, and $85.7 \%$ to $97.0 \%$, respectively). The Lancet needle had the lowest sensitivity, NPV, and diagnosis accuracy rate in only histology, but when cytology was combined, the results were similar to those of other needles. EUS-guided tissue acquisition is a sufficiently useful examination when done by both experts and trainees even in cases of small solid pancreatic lesions.

Recently, small solid pancreatic lesions have been increasingly found due to advances in diagnostic imaging [5]. However, if a lesion is small, it is sometimes difficult to diagnose with imaging alone. Pancreatic cancer is the most common type of solid pancreatic lesion. A diameter of less than $2 \mathrm{~cm}$ has been reported as a better prognostic factor compared to a diameter greater than $2 \mathrm{~cm}$ [11]. Therefore, it is important to make a precise diagnosis at this stage. Benign tumors can sometimes be confused with small malignant tumors. However, it is difficult to observe these tumors with only diagnostic imaging when malignancy cannot be ruled out. Therefore, a precise pathological diagnosis is required even for small solid pancreatic lesions.

Three types of needles were analyzed in this study. Recently, several studies reported the usefulness of Franseen needles in diagnosis of solid pancreatic lesions $[12,13]$. The accuracy in the Franseen group in this study tended to be low. There is a difference in the shape of the needle tip compared to the shapes of the other needles that may affect the results. The tips of the Lancet and the Menghini needle are sharp end-cut types that can easily puncture the target site. On the other hand, the tip of the Franseen needle consists of three symmetrical cutting surfaces with formed heels to obtain adequate amounts of tissue into the needle. There is a possibility that the resistance of the Franseen needle with unique tip will be greater than that of the other two needles. We found that the lesion moves in conjunction with the Franseen needle, so it is sometimes impossible to get a sufficient stroke, especially within small lesion. The Lancet and Menghini needles may make it easier to pull from within small lesions efficiently due to the good needle advancement. The results of histological diagnosis alone between each needle were not statistically significant, but the Menghini needle showed a trend toward higher NPVs among these three nee- dles. A high NPV can be considered an advantage in preventing excessive treatment, but the results should be interpreted with caution due to the low number of benign diseases. Histological diagnosis is also important to distinguish pancreatic cancer from neuroendocrine neoplasms. Several studies have reported that EUS-FNB is more useful in diagnostic yield than EUS-FNA [13-19]. However, there are only a few reports on use of EUSFNB in small solid pancreatic lesions. The Menghini needle may be useful for puncturing small solid pancreatic lesions. However, lesions less than $1 \mathrm{~cm}$ in diameter may not be good candidates for using the Menghini needle because of the lateral groove at its tip.

The frequency of AEs observed following EUS-guided tissue acquisition for solid pancreatic lesions was approximately $0 \%$ to $2 \%[14,17,20]$. However, there are few reports of AEs for small solid pancreatic lesions. Chrino S.F. et al reported that pancreatitis occurred after EUS-guided tissue acquisition for solid pancreatic lesions of less than $15 \mathrm{~mm}$ diameter in two of 61 cases (3.3\%) [4]. EUS-guided tissue acquisition for small pancreatic lesions is likely to be associated with a high risk of pancreatitis [21] because the pancreatic parenchyma is frequently present in the puncture line, increasing risk of pancreatic duct injury, and pancreatic atrophy is poor without obstruction of the main pancreatic duct in small solid pancreatic lesions. In this study, AEs occurred in four cases (2.5\%), including two patients with pancreatitis. To reduce AEs, we need to avoid accidental puncture of the main pancreatic duct, shorten the puncture line through the normal pancreatic parenchyma, and decrease the number of punctures.

There are several limitations to this study. The first that it was retrospective and performed at a single center. However, there is no other report comparing needles with different shapes in terms of diagnostic yield for small solid pancreatic lesions. The second limitation is that there were more punctures and larger lesions and puncture diameters were observed in the Menghini group compared to the other two groups, which may have influenced the good diagnostic yield in the Menghini group. However, the Menghini group only had one additional puncture and puncture diameters that were only 1 to $2 \mathrm{~mm}$ larger, which are not large differences. The third limitation is that we focused only on solid pancreatic lesions, so it is unclear whether this result can be applied to submucosal tumors or intraabdominal lesions in locations such as the lymph nodes. The fourth limitation is that lesion size was measured with EUS and EUS cannot necessarily detect the maximal aspect of a lesion. Finally, because the amount of collected tissue was not measured, it was not possible to determine exact differences in the amounts of tissue collected by each needle.

\section{Conclusion}

In conclusion, EUS-guided tissue acquisition for solid pancreatic lesions less than $2 \mathrm{~cm}$ in diameter has a high diagnostic yield and is safe. This study showed a difference in the diagnostic yield for each needle. Further evaluation is needed, in a prospective randomized study, to confirm the difference in diagnostic yield among the needles. 


\section{Competing interests}

TS has received honoraria from Boston Scientific Japan, Cook Medical Japan and Century Medical Inc. NS has received honoraria from Boston Scientific Japan and Cook Medical Japan. The other authors declare no conflict of interest.

\section{References}

[1] Vilmann P, Jacobsen GK, Henriksen FW et al. Endoscopic ultrasonography with guided fine needle aspiration biopsy in pancreatic disease. Gastrointest Endosc 1992; 38: 172-173

[2] Itoi T, Sofuni A, Itokawa F et al. Current status of diagnostic endoscopic ultrasonography in the evaluation of pancreatic mass lesions. Dig Endosc 2011; 23: 17-21

[3] Dumonceau JM, Deprez PH, Jenssen C et al. Indications, results, and clinical impact of endoscopic ultrasound (EUS)-guided sampling in gastroenterology: European Society of Gastrointestinal Endoscopy (ESGE) Clinical Guideline - Updated January 2017. Endoscopy 2017; 49: 695-714

[4] Crino SF, Conti Belocchi MC, Bernardoni L et al. Diagnostic yield of EUS-FNA of small $(\leq 15 \mathrm{~mm})$ solid pancreatic lesions using a 25 -gauge needle. Hepatobiliary Pancreat Dis Int 2018; 17: 70-74

[5] Egawa S, Toma H, Ohigashi H et al. Japan Pancreatic Cancer Registry; 30th Year Anniversary: Japan Pancreas Society. Pancreas 2012; 41: 985-992

[6] Nakai Y, Isayama H, Chang KJ et al. Slow pull versus suction in endoscopic ultrasound-guided fine-needle aspiration of pancreatic solid masses. Dig Dis Sci 2014; 59: 1578-1585

[7] Cotton PB, Eisen GM, Aabakken L et al. A lexicon for endoscopic adverse events: report of an ASGE workshop. Gastrointest Endosc 2010; 71: $1446-1454$

[8] Kanda Y. Investigation of the freely available easy-to-use software 'EZR' for medical statistics. Bone Marrow Transplant 2013; 48: 452458

[9] Ali A, Siddiqui L], Brown SKS et al. Relationship of pancreatic mass size and diagnostic yield of endoscopic ultrasound-guided fine needle aspiration. Dig Dis Sci 2011; 56: 3370-3375

[10] Carlo F, Carmelo L, Antonella M et al. Endoscopic ultrasound-guided fine-needle biopsy of small solid pancreatic lesions using a 22-gauge needle with side fenestration. Surg Endosc 2015; 29: 1586-1590

[11] Japan Pancreas Society. General Rules for the Study of Pancreatic Cancer. 7th ed. 2016
[12] Mitri RD, Rimbas M, Attili F et al. Performance of a new needle for endoscopic ultrasound-guided fine-needle biopsy in patients with pancreatic solid lesions: A retrospective multicenter study. Endosc Ultrasound 2018; 7: 329-34

[13] Ihab I, El H, Howard W et al. Prospective asessment of the performance of a new fine needle biopsy device for EUS-guided sampling of solid lesions. Clin Endosc 2018; 51: 576-583

[14] Fujie S, Ishiwatari H, Sasaki K et al. Comparison of the diagnostic yield of the standard 22-gauge needle and the new 20-gauge forwardbevel core biopsy needle for endoscopic ultrasound-guided tissue acquisition from pancreatic lesions. Gut Liver 2019; 13: 349-355

[15] van Riet P, Alberto L, Fabia A et al. A multicenter randomized trial comparing a 25-gauge EUS fine-needle aspiration device with a 20gauge EUS fine-needle biopsy device. Gastrointest Endosc 2019; 89: 329-339

[16] Nishioka N, Ogura T, Kurisu Y et al. Prospective histological evaluation of a 20G core trap with a forward-cutting; bevel needle for EUS-FNA of pancreatic lesions. Surg Endosc 2018; 32: 4125-4131

[17] Elia A, Erminia M, Elena T et al. Histologic retrieval rate of a newly designed side-bevelled 20G needle for EUS-guided tissue acquisition of solid pancreatic lesions. United Europ Gastroenterol J 2019; 7: 96104

[18] Mukai S, Itoi T, Yamaguchi $\mathrm{H}$ et al. A retrospective histological comparison of EUS-guided fine-needle biopsy using a novel Franseen needle and a conventional end-cut type needle. Endosc Ultrasound 2019; 8: 50-57

[19] Bang JY, Kirtane S, Krall K et al. In memoriam: Fine-needle aspiration, birth: Fine-needle biopsy: The changing trend in endoscopic ultrasound-guided tissue acquisition. Dig Endosc 2019; 31: 197-202

[20] Ryozawa S, Kioh H, Komaki T et al. Usefulness of endoscopic ultrasound-guided fine-needle aspiration biopsy for the diagnosis of pancreatic cancer. J Gastroenterol 2005; 40: 907-911

[21] Eloubeidi MA, Gress FG, Savides TJ et al. Acute pancreatitis after EUSguided FNA of solid pancreatic masses: a pooled analysis from EUS centers in the United States. Gastrointest Endosc 2004; 60: 385-389

\section{CORRECTION}

Takafumi Mie, Takashi Sasaki, Ryo Kanata et al. Diagnostic yield of endoscopic ultrasound-guided tissue acquisition for small solid pancreatic lesions

Endoscopy International Open 2020; 08: E1359-E1364.

DOI: $10.1055 / a-1230-3555$

The article was published with the incorrect corresponding author and affiliations.

The corresponding author is:

Takashi Sasaki, Cancer Institute Hospital of Japanese Foundation for Cancer Research, Department of Gastroenterology Center, Division of Hepato-Biliary-Pancreatic Medicine, 3-8-31 Ariake, Koto-ku, Tokyo 135-8550, Japan Fax: +81335200141 sasakit-tky@umin.ac.jp

The affiliation of all authors are: Cancer Institute Hospital of Japanese Foundation for Cancer Research, Department of Gastroenterology Center, Division of Hepato-Biliary-Pancreatic Medicine, Tokyo, Japan 